\title{
Clino-San Vaginal Lubricant
}

National Cancer Institute

\section{Source}

National Cancer Institute. Clino-San Vaginal Lubricant. NCI Thesaurus. Code C74018.

A vaginal lubricant formulated as a topical gel used to prevent and treat vaginal dryness.

With a pH similar to that of normal vaginal discharge, Clino-San vaginal lubricant may

reduce dryness, pain and irritation often caused by insufficient vaginal discharge due to atrophy of the vulvovaginal mucosa. 